\title{
Supplementary Data of
}

Mechanistic Study of Potent Fluorinated EGFR Kinase Inhibitors with a Quinazoline Scaffold against L858R/T790M/C797S Resistance Mutation: Unveiling the Fluorine Substituents Cooperativity Effect on the Inhibitory Activity

Farideh Badichi Akher ${ }^{\mathrm{a}, \mathrm{b}^{*}}$, Abdolkarim Farrokhzadeh ${ }^{\mathrm{c}}$, Neil Ravenscroft ${ }^{\mathrm{b}}$ and Michelle M. Kuttel ${ }^{a^{*}}$

a Department of Computer Science, University of Cape Town, Cape Town, South Africa

${ }^{b}$ Department of Chemistry, University of Cape Town, Cape Town, South Africa

c School of Chemistry and Physics, University of KwaZulu-Natal, Private Bag X01, Pietermaritzburg 3209, South Africa

*Corresponding Authors: Farideh Badichi Akher, Michelle M. Kuttel

Email addresses: farideh.badichiakher@uct.ac.za, mkuttel@cs.uct.ac.za

Figure S1. Molecular topography of 25g-F856 dimer obtained from representative snapshot for $\mathrm{EGFR}^{\mathrm{TM}}-\mathbf{2 5 g}$. The small green and red spheres correspond to bond critical points and ring critical points, respectively. 


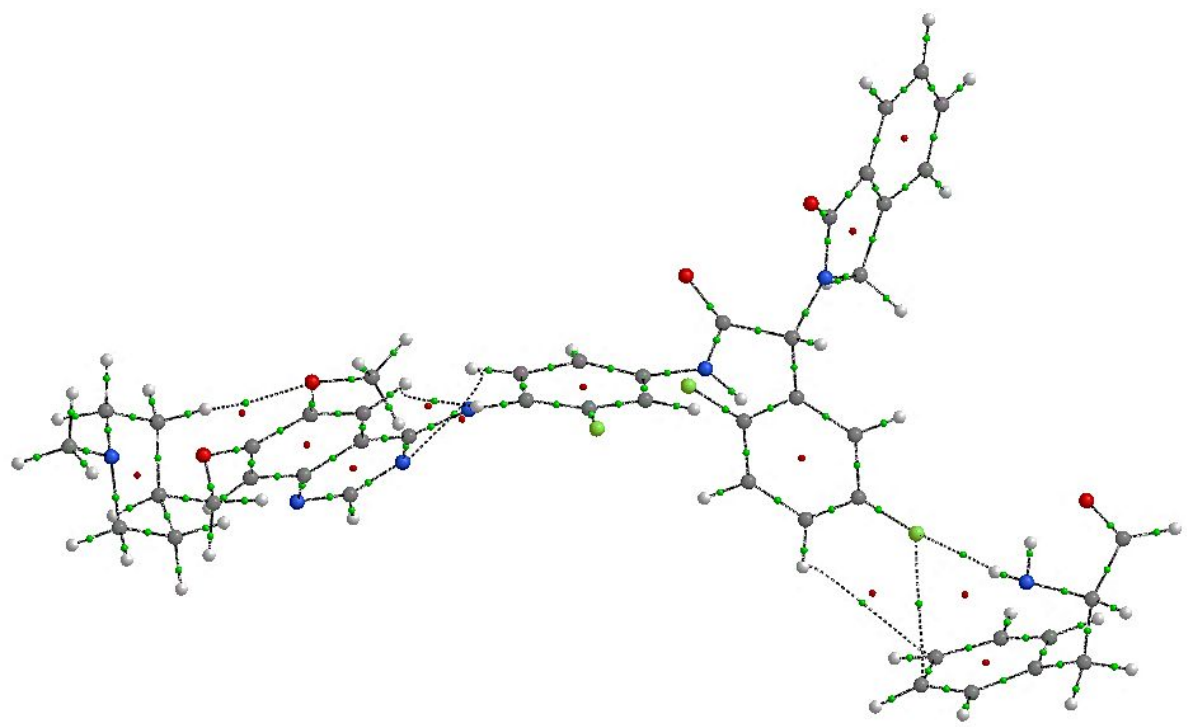

Figure S1. Molecular topography of 25g-F856 dimer obtained from representative snapshot for EGFR $^{\mathrm{TM}}$-25g. The small green and red spheres correspond to bond critical points and ring critical points, respectively. 